J Neurosurg 121:498-500, 2014

(C)AANS, 2014

\title{
Erratum
}

\section{Hockey Concussion Education Project, Part 2. Microstructural white matter alterations in acutely concussed ice hockey players: a longitudinal free-water MRI study}

\author{
Clinical article
}

To The Editor: We appreciate publication of our paper entitled "Hockey Concussion Education Project, Part 2. Microstructural white matter alterations in acutely concussed ice hockey players: a longitudinal free-water MRI study. Clinical article" (J Neurosurg 120:873-881, 2014). After our paper had been published, an error in the analysis was identified. We would like to correct the record at this time. Specifically, we mislabeled some of the post-concussion subjects. Correct labeling of these subjects modified the group differences that we reported in the text and figures, and the statistical significance of most reported parameters was thereby improved. In addition to our previously reported findings, we now report significant decreases in FAt, but we no longer report significant decreases in RDt.

Our revised analysis does not affect the conclusions of the paper. In particular, we are making the following corrections, which are displayed in boldface text in the following sections:

\begin{abstract}
1. Results, first sentence: "Paired comparison of the concussed players before and after injury revealed a statistically significant $(\mathrm{p}<0.05)$ common pattern of reduced free-water volume and reduced axial diffusivity and fractional anisotropy following elimination of free-water."

2. Conclusions, third sentence: "This finding might be explained by axonal injury and/or by increased cellularity of glia cells."
\end{abstract}

\section{Text of Article}

3. Results section, Atlas-Based Analysis subsec-

Abbreviations used in this erratum: $\mathrm{ADt}=$ axial diffusivity corrected for free-water; FA = fractional anisotropy; FAt $=$ FA corrected for free-water; $\mathrm{RD}=$ radial diffusivity; $\mathrm{RDt}=\mathrm{RD}$ corrected for free-water.

Please include this information when citing this paper: published online June 27, 2014; DOI: 10.3171/2014.5.JNS132090a. tion, second paragraph, second through fourth sentences: "This comparison revealed the following statistically significant changes following concussion (Fig. 3): increased FA $(\mathrm{t}=\mathbf{2 . 1 6} ; \mathrm{p}=\mathbf{0 . 0 3 6 9})$, decreased free-water $(\mathrm{t}=\mathbf{3 . 1 5}$; $\mathrm{p}=\mathbf{0 . 0 0 9 9})$, decreased ADt $(\mathrm{t}=\mathbf{3 . 3 8} ; \mathrm{p}=\mathbf{0 . 0 0 7 4})$, and decreased FAt $(t=2.27 ; p=0.0319)$. Increases in FAt were not significant $(\mathrm{t}=\mathbf{1 . 1 6} ; \mathrm{p}=\mathbf{0 . 1 4 5 0})$. None of the extent measures correlated with the number of days between the first and second assessments."

4. Results section, Atlas-Based Analysis subsection, third paragraph, third sentence: "Figure 5 shows, for each individual, the changes in the extent measure of decreased ADt, decreased FAt, and decreased free-waterthe 3 measures that had a significant change following concussion."

5. Discussion section, third paragraph, first sentence: "Using free-water elimination and comparing tensor indices obtained from the tissue compartment, we identified alterations in the tissue vicinity affecting FAt and ADt."

6. Discussion section, fourth paragraph, first through third sentences in the print version (first and second sentences in the corrected online version): "The decrease in FAt likely reflects the decrease in ADt and may be related to axonal injury. ${ }^{38}$ On the other hand, decreased ADt and a decrease in the free-water measure could be due to an increased number of glial cells."

7. Discussion section, fourth paragraph, seventh through ninth sentences in the print version (sixth and seventh sentences in the corrected online version): "We therefore suggest that the ADt, FAt, and free-water changes that we observed might be connected to both processes: axonal injury and increased cellularity. We note that increased cellularity might be a normal response of the immune system, ${ }^{8,39}$ and further studies would be required to determine whether these changes lead to long-term symptoms or not."

This article contains some figures that are displayed in color online but in black-and-white in the print edition. 

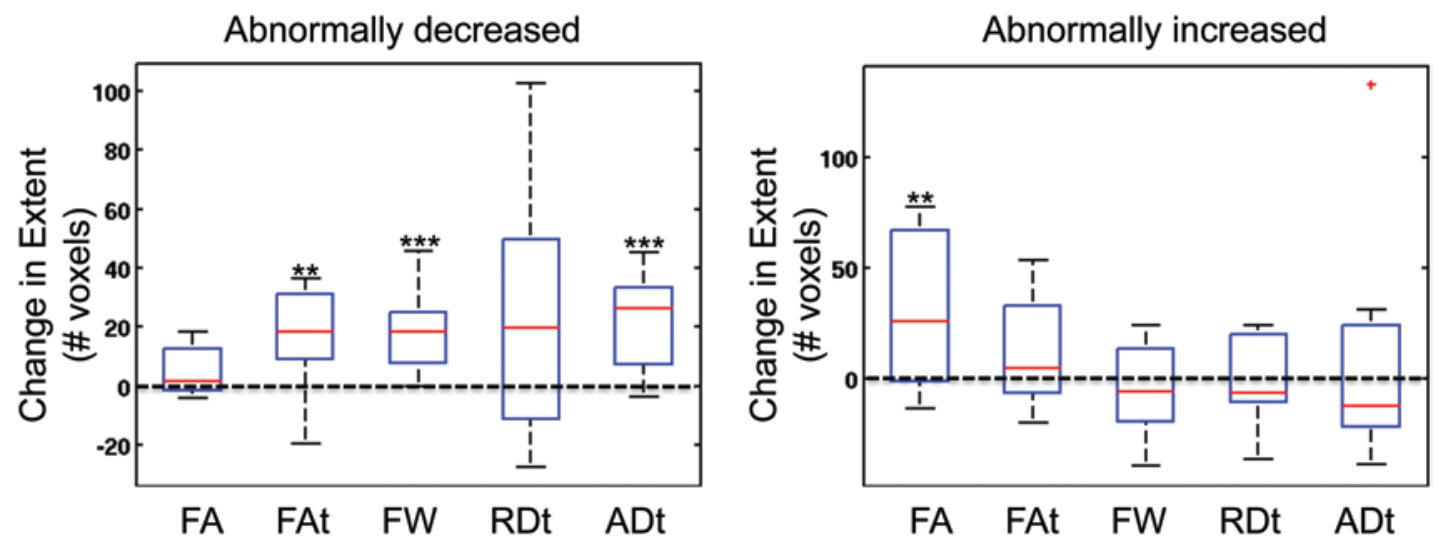

FIG. 3. Comparison of extent measures. Comparing the extent measure before and after a concussion shows that there were more abnormally decreased free-water, FAt, and ADt voxels after a concussion than before a concussion. In addition, there were more voxels with abnormally increased FA following the concussion, although following a free-water correction the FAt measure was not significantly increased. ${ }^{* *} p<0.05 ;{ }^{* * *} p<0.01$.

8. Discussion section, sixth paragraph, fourth sentence: "This might explain the reduction in the extent measure that was evinced in a small number of players following concussion."

\section{Figures}

9. Figures 3, 4, and 5. The plots in these figures have been changed. The figure legends have been updated with boldface text showing the changes.
We apologize to the editor and readers for these errors, and we are pleased to have the opportunity to make these corrections. The corrections were made as of June $27,2014$.

Ofer Pasternak, Ph.D. Martha Shenton, Ph.D. Harvard Medical School Boston, MA Paul Echlin, M.D. The Elliott Sports Medicine Clinic Burlington, ON, Canada

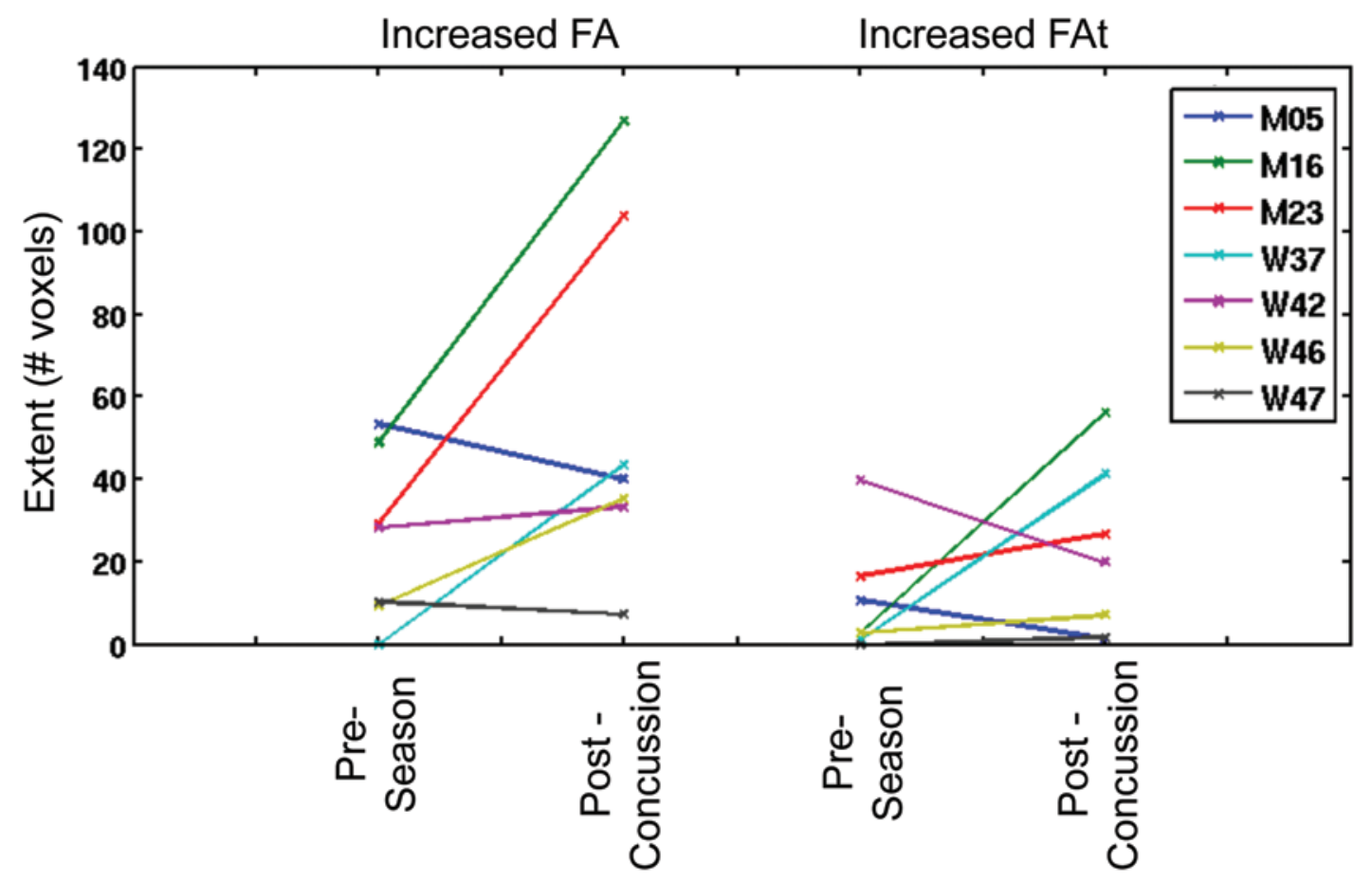

FIG. 4. Individual differences in increased FA and increased FAt. There were large changes (mainly increases) in the extent of increased FA following the concussion. However, these changes were not evident in the extent of increased FAt, suggesting that the free-water correction accounted for some of the differences evinced in the FA measure. This means that extracellular changes may have contributed to the anisotropy changes. $M=$ male; $F=$ female. 
Erratum

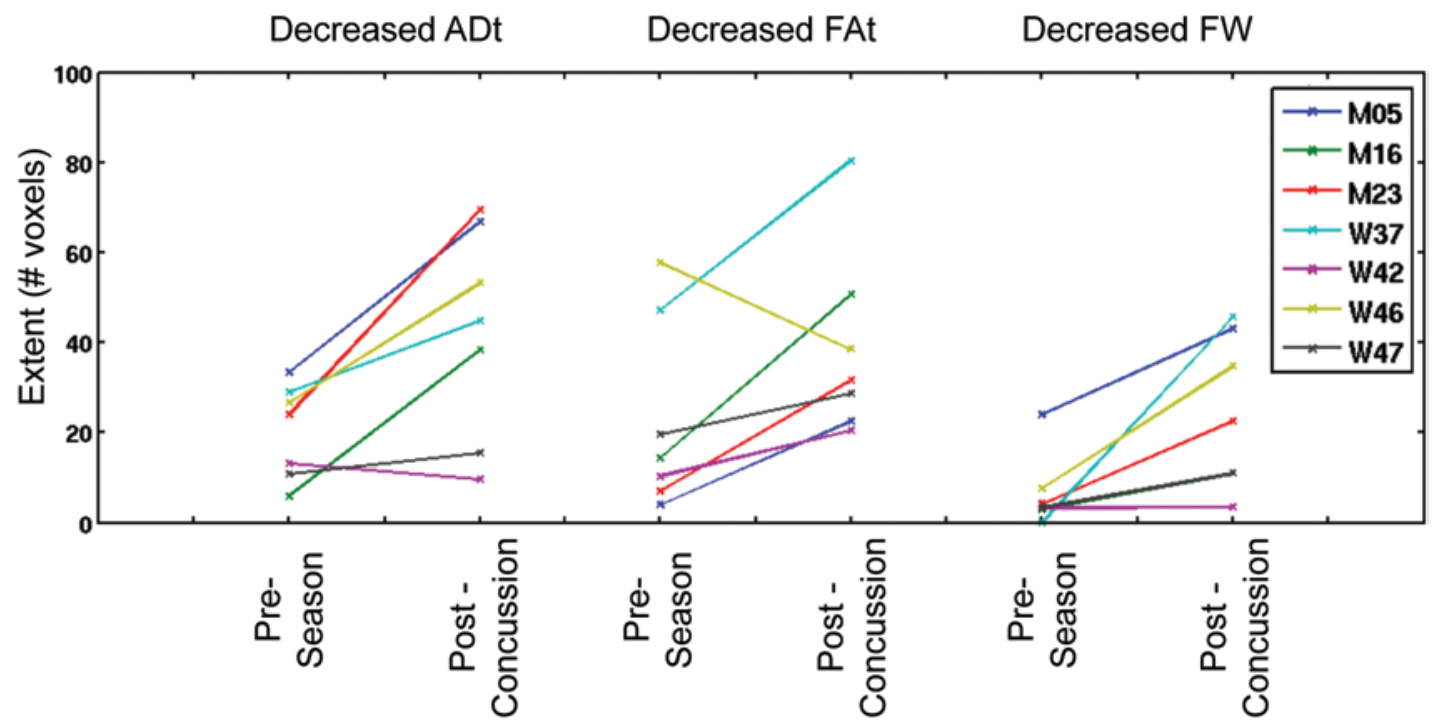

FIG. 5. Individual differences in ADt, FAt, and the free-water measure (FW). The decreases in the extent of ADt, RDt, and FW were consistent in the 7 concussed players. The degree of change varied across the players. 